\title{
Are tutor-students capable of writing good biochemistry exams?
}

\author{
Alexandre B. Sé; Renato M. Passos; Marcelo Hermes-Lima
}

Depto. Biologia Celular, UnB, Brasília, DF, 70910-900

In a previous article we described the relevance of student seminars for the learning process of applied biochemistry for medical and nutrition students (Hermes-Lima et al., Biochem. Mol.Biol.Educ. 30: 30-34,2002). First semester students of a basic biochemistry course (BioBio) are divided in 10 groups of 5 members, and each group is assigned to a specific topic (diabetes, cholesterol, etc) under the supervision of a tutor-student. The tutors have already coursed BioBio and are currently undertaking an advanced biochemistry course. In order to evaluate the learning of applied biochemistry for BioBio students a true or false exam (TFE) is performed. This exam is made of 50 questions ( 5 on each topic) elaborated by the tutors under the supervision of the teacher. The TFE corresponds to 10 percent of the grade of BioBio and focus on clinical and/or applied biochemistry situations. At the end of the exam, BioBio students were asked to share their opinions about TFEs $(\mathrm{n}=401$, from $2001 / 1$ to $2003 / 2$ ). When asked to give a 0-to-4 score regarding (a) the difficulty level of the test, (b) the technical quality and (c) if the exam makes an appropriate evaluation of applied biochemistry knowledge, the scores were 2.9, 3.4 and 2.9, respectively. BioBio students were also asked if they find valid to be evaluated by a tutor-made exam and if they would like to participate in the making of TFEs; 96 and 58 percent answered yes, respectively.

In another survey, we interviewed former BioBio students from the 2 nd to the 7 th semesters $(n=95)$ about TFEs (since 1999-1) regarding technical aspects, which included (1) clarity of questions, (2) level of difficulty, (3) clinical application and (4) thinking (as opposed to memorizing) abilities demanded; the 0 -to-4 scores were $3.1,2.9,2.6$, and 2.5 , respectively. Other four questions were on the validity of tutors writing TFEs and their capacity to perform such a task; the average score was 3.2. Our surveys show the students good acceptance of the seminar system (Hermes-Lima et al. 2002), of the TFEs-based evaluation system, and students interest in TFEs writing (tutors also enjoy the process). These positive aspects constitute a motivation on the learning of basic biochemistry.

Acknowledgments: CNPq, FM-UnB and about 100 former tutors. 